Supporting Information

\title{
Electromechanically Actuated MXene Nanotubes for Tunable Mass Transport
}

Bozhao $\mathrm{Wu}^{1 \#}$, Yuanpeng $\mathrm{Yao}^{1 \#}, \mathrm{Ke} \mathrm{Zhou}^{2}, \mathrm{Ze} \mathrm{Liu}^{1 *}$, and Enlai Gao ${ }^{1 *}$

${ }^{1}$ Department of Engineering Mechanics, School of Civil Engineering, Wuhan University, Wuhan, Hubei 430072, China.

${ }^{2}$ State Key Laboratory for Strength and Vibration of Mechanical Structures, School of Aerospace Engineering, Xi'an Jiaotong University, Xi'an 710049, China.

\#These authors contribute equally.

*Corresponding author. Email: ze.liu@whu.edu.cn; enlaigao@whu.edu.cn 
(a)
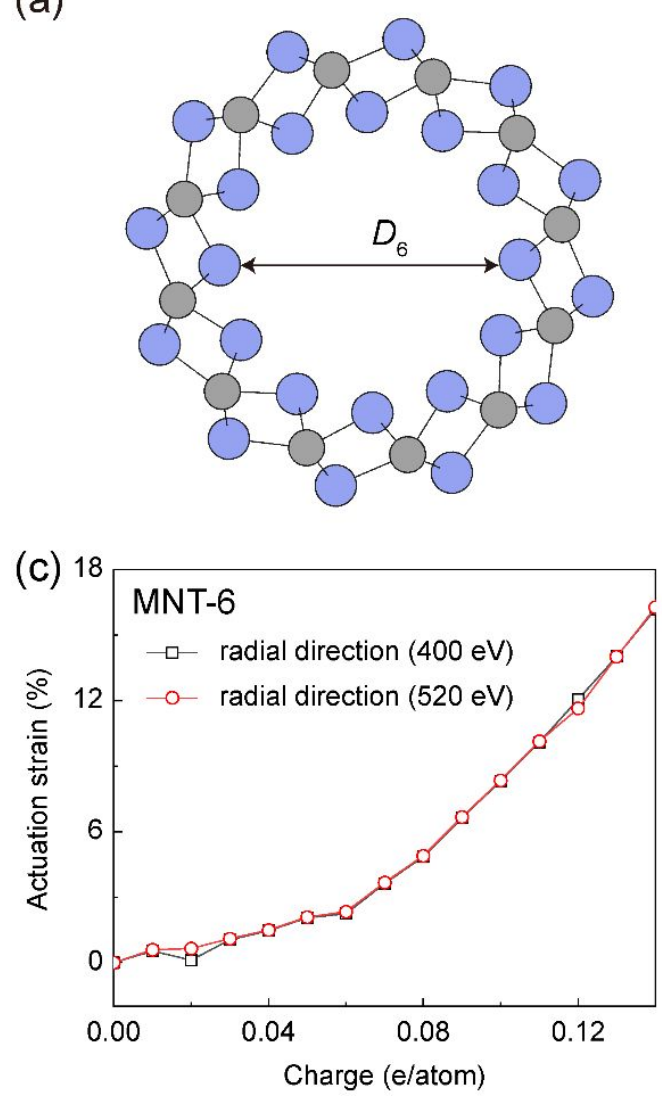

(b)
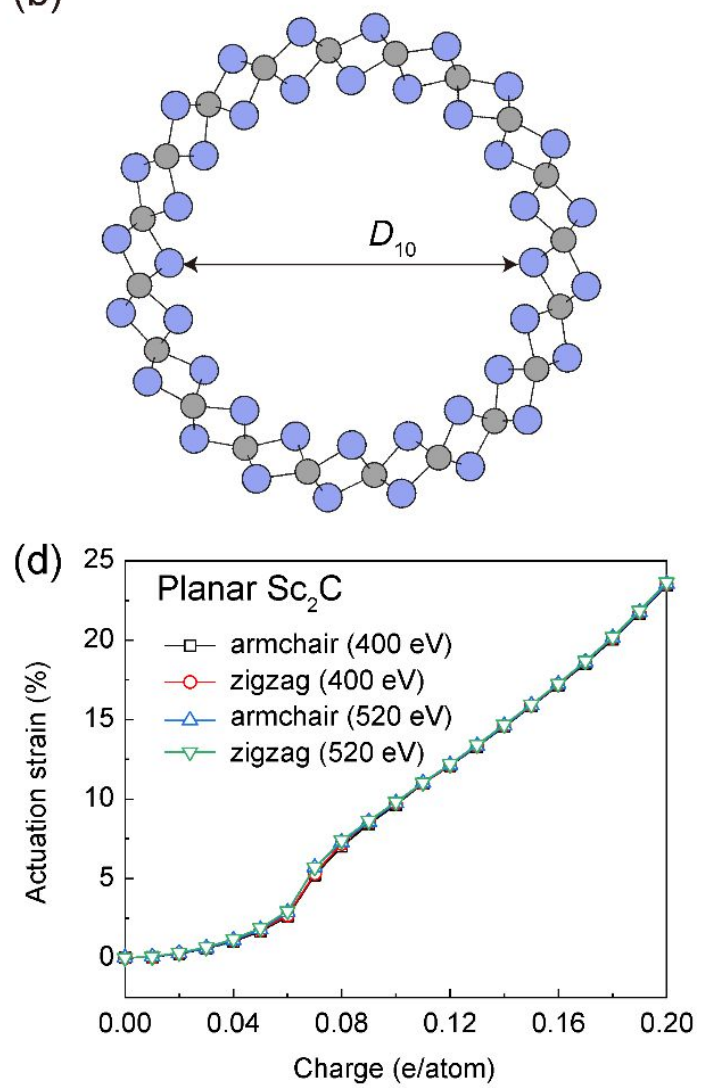

Figure S1. (a-b) Atomic configurations of (a) MNT-6 and (b) MNT-10. (c-d) Convergence tests for the energy cutoff. Evidently, the calculated actuation strains of planar $\mathrm{Sc}_{2} \mathrm{C}$ and MNT-6 using an energy cutoff of 400 $\mathrm{eV}$ are almost same to that using an energy cutoff of $520 \mathrm{eV}$, indicating that the energy cutoff of $400 \mathrm{eV}$ provides a good balance between computational accuracy and effort. 

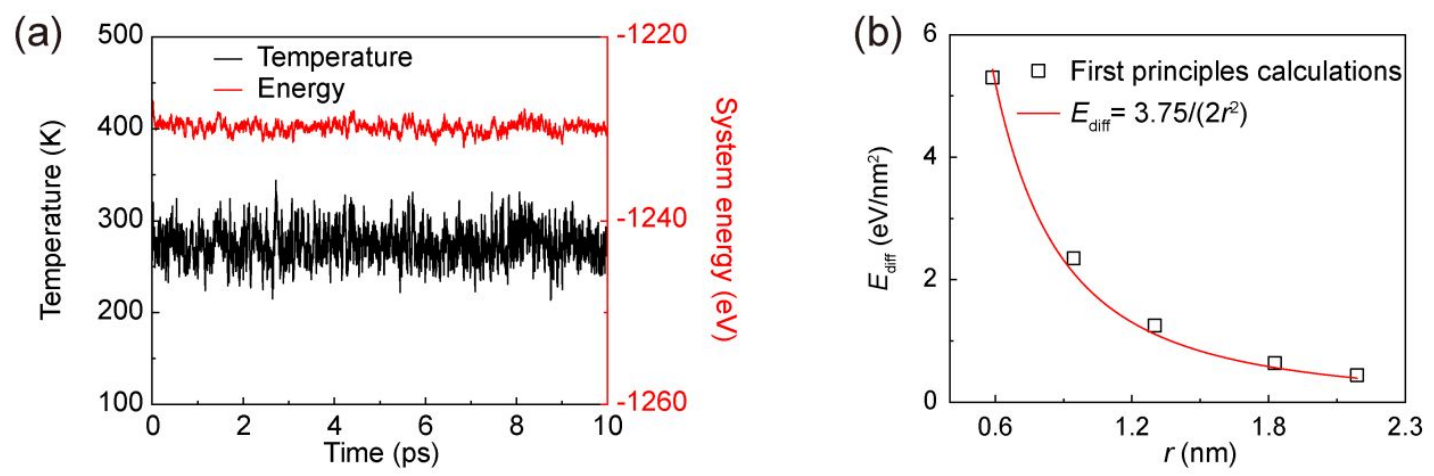

Figure S2. (a) Evolutions of temperature and system energy during AIMD simulations, which were performed at $298 \mathrm{~K}$ for $10 \mathrm{ps}$ using Andersen thermostat to control the temperature (see Movie S1 for the animation). (b) Energy per $\mathrm{nm}^{2}$ versus the radius $(r)$ (herein, $r$ is the radius of circular $\mathrm{Sc}_{2} \mathrm{C}$ ): first principles calculations data (symbols) and continuum model (red solid lines, with a bending stiffness of $3.75 \mathrm{eV}$ ). 
(a)

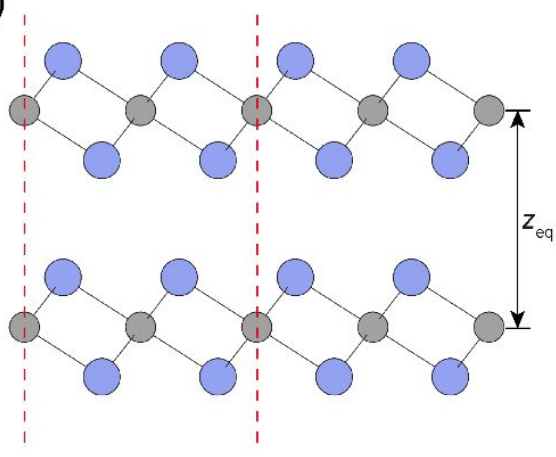

(c)

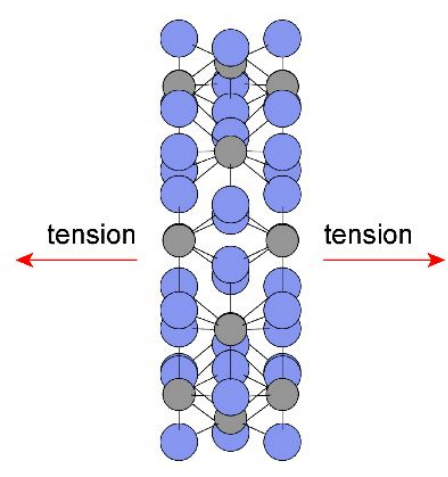

(b)

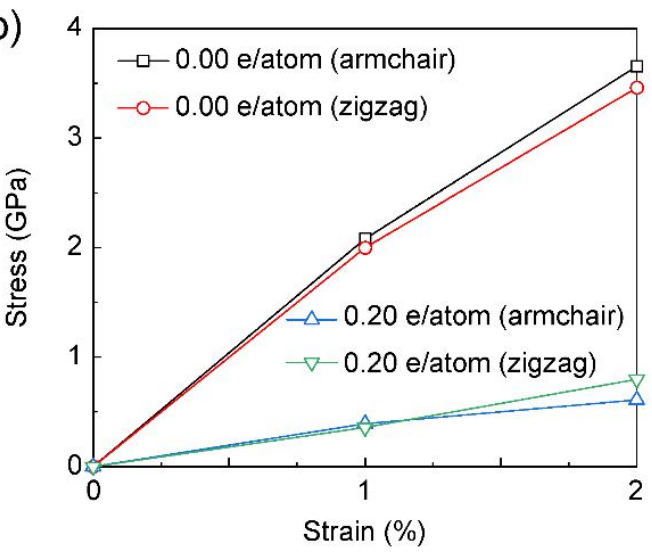

(d)

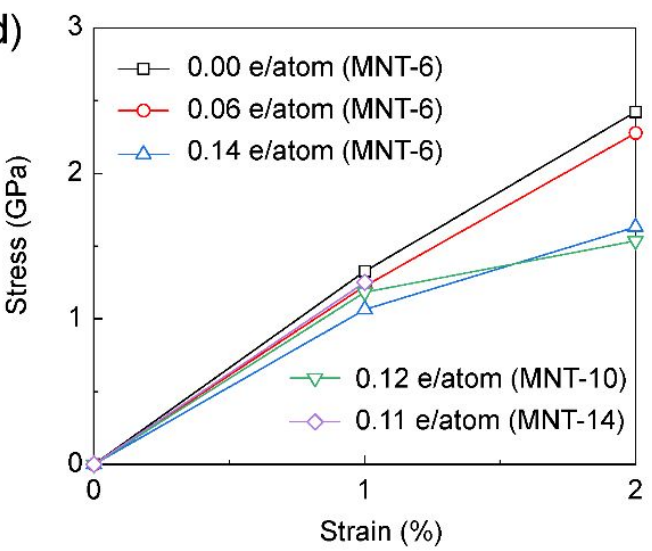

Figure S3. Structural integrity under combined electromechanical loads. (a) Bilayer $\mathrm{Sc}_{2} \mathrm{C}$ was used to determine the interlayer spacing $\left(z_{\text {eq }}\right)$. The unit cell is within the red dashed lines. (b) Tensile tests of planar $\mathrm{Sc}_{2} \mathrm{C}$ upon charge injection of 0.20 e/atom. (c) Illustration of MNTs under the tension. (d) Tensile tests of MNTs upon charge injection. 

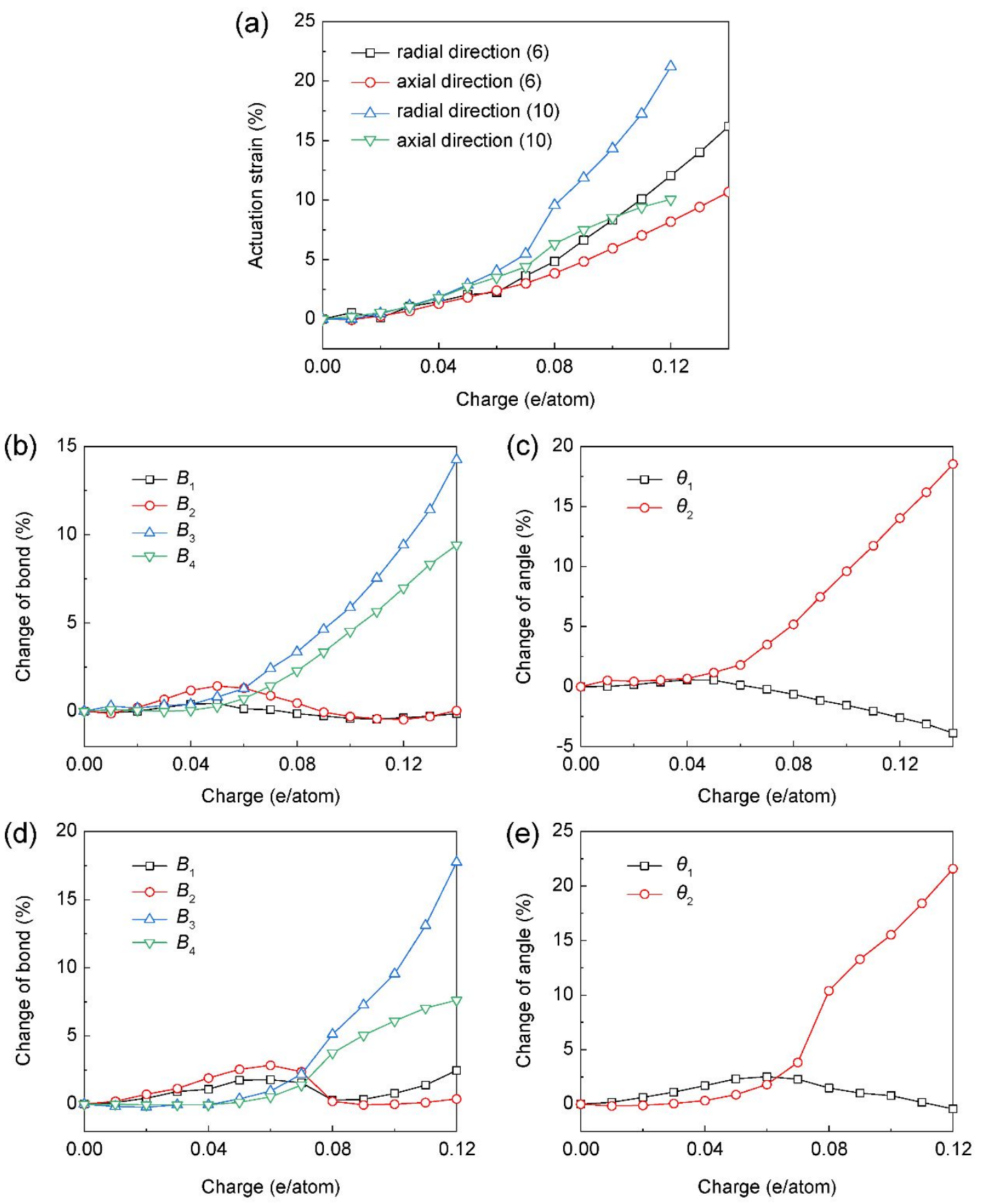

Figure S4. (a) Actuation performance of MNT-6 and MNT-10 upon charge injection. (b-e) Corresponding evolutions of the change of interatomic parameters of MNT-6 and MNT-10 as functions of charge injection. Changes of bond lengths $\left(B_{1-4}\right)$ and angles $\left(\theta_{1-2}\right)$ of (b-c) MNT-6 and (d-e) MNT-10 upon charge injection. 
(a)
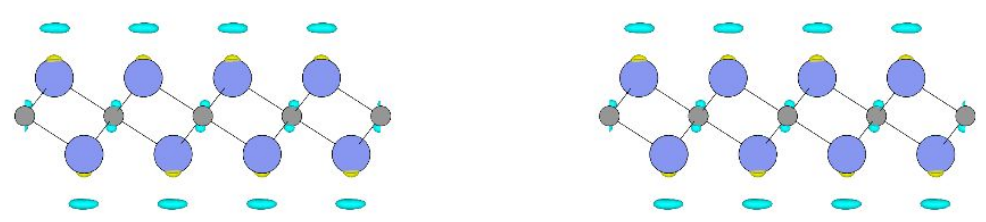

(b)

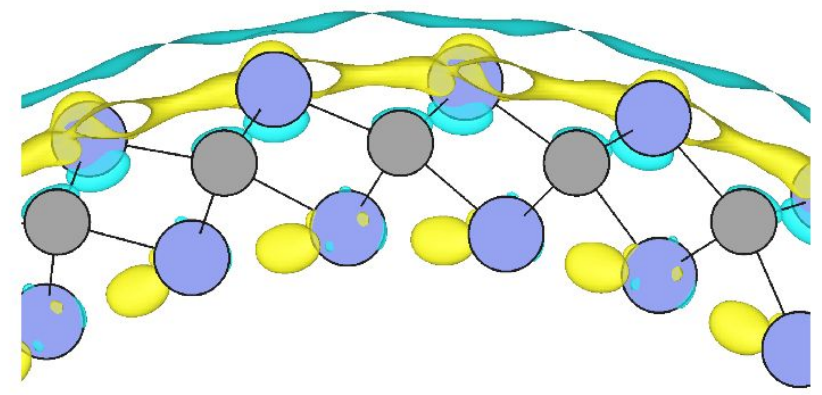

Figure S5. (a) Excess charge density distributions of (a) planar $\mathrm{Sc}_{2} \mathrm{C}$ and (b) MNT-14 upon 0.01 e/atom injection with the iso-surface value of 0.00015 and $0.00009 \mathrm{e} / \mathrm{Bohr}^{3}$, respectively. The yellow and blue colors represent excess electron and hole, respectively. 
Supporting Information Movie 1:

AIMD simulations of MNT-14 at $298 \mathrm{~K}$ for 10 ps.

Supporting Information Movie 2:

MNT-14 upon charge injection. 